\title{
Improvement of Factory Planning by Automated Generation of a Digital Twin
}

\author{
Markus SOMMER ${ }^{\mathrm{a}}$, Josip STJEPANDIĆ ${ }^{\text {, }}$, Sebastian STOBRAWA ${ }^{\mathrm{c} 1}$, \\ and Moritz VON SODEN ${ }^{\mathrm{d}}$ \\ ${ }^{a}$ isb - innovative software business GmbH, Germany \\ ${ }^{b}$ PROSTEP AG, Germany \\ ${ }^{c}$ Leibniz University Hannover, Institute of Production Engineering and Machine Tools, \\ Germany \\ ${ }^{d}$ Bornemann Gewindetechnik GmbH \& Co. KG
}

\begin{abstract}
The simulation of processes in production systems is a powerful tool for factory planning. The application of simulation methods within the Digital Factory is becoming increasingly relevant as developments in the field of digitalization lead to more comprehensive, efficient, embedded and cost-effective simulation methods. Especially the integration within a Digital Twin, allows these advantages to be achieved for simulations. Here, the Digital Twin can be utilized for prospective planning, analysis of existing systems or process-oriented monitoring. In all cases, the Digital Twin offers manufacturing companies room for improvement in production and logistics processes leading to cost savings. However, many companies do not apply the technology, because the generation of a Digital Twin is cost-, time- and resource-intensive and IT expertise is required. This paper presents an approach for generating a Digital Twin in the built environment automatically and for utilization in factory planning. The obstacles will be overcome by using a scan of the shop floor, subsequent object recognition, and predefined frameworks for factory planning within the Digital Twin. Here, the effort for scanning the production hall is additional, while the subsequent object recognition, the generation of the CAD model and the simulation model, as well as the factory planning can be to a great extent automated and therefore carried out with a minimum of effort. Therefore, considerable cost savings can be expected here, which more than offset the additional effort for scanning.
\end{abstract}

Keywords. Factory Planning, Digital Twin, Object Recognition, Process Template

\section{Introduction}

Since the digital factory has been recognized as a strategically competitive advantage by the industry, small and medium-sized enterprises as well as large companies are increasingly focusing on digital factory methods and tools [1]. In particular, simulation is a core element of the digital factory and is becoming more important as a result of developments in the area of digitalization [2]. In the field of production and logistics, simulation has been scientifically investigated and established for a long time [3][4][5]. Thus, it supports companies in optimizing logistical targets (e. g. with regard to

\footnotetext{
${ }^{1}$ Corresponding Author, Mail: stobrawa@ifw.uni-hannover.de.
} 
adherence to schedules, throughput times, and costs), as well as in the tasks of material flow planning [6][7].

Today, there are many fields of application for digital models of a production system in a discrete event simulation (DES), such as layout optimization in the shop floor, planning of factories and optimization of production processes. Nevertheless, current studies prove that the use of simulation models for production systems, referred to as Digital Twin, in small and medium-sized enterprises is still not standard [8]. The main reasons for this are non-transparent procurement costs, required IT expertise, unpredictable operating expenses and lack of knowledge regarding available simulation tools and application areas as well as the achievable benefits [8][9][10]. In order to overcome these obstacles, an approach is introduced here, which allows a preferably automated generation of a Digital Twin by an intelligent workflow.

The remainder of this paper is structured as follows: Section 1 provides an insight into literature review, followed by section 2, where the parameters for the Digital Twin are discussed. A new concept for simulation modeling and factory planning is described in section 3. The results are presented in section 4 , followed by conclusions and outlook in section 5 .

\section{Background}

Industry 4.0, cyber-physical production systems (CPPS) and the Internet of Things (IoT) are current focusses in automation and data exchange in manufacturing, arising from the rapid increase in capabilities in information and communication technologies and the ubiquitous internet. A key enabler for the advances promised by CPPS is the concept of a Digital Twin, which is the virtual representation of a real-world entity, or the physical twin. An important step towards the success of Industry 4.0 is the establishment of practical reference architectures. An architecture for such a Digital Twin, which enables the exchange of data and information between a remote emulation or simulation and the physical twin is presented. The architecture comprises different layers, including a local data layer, an IoT Gateway layer, cloud-based databases and a layer containing emulations and simulations. The architecture can be implemented in new and legacy production facilities, with a minimal disruption of current installations. This architecture provides a service-based and real-time enabled infrastructure for vertical and horizontal integration. An intelligent workflow is an important means for implementation of such infrastructures [11].

By using a Digital Twin, enterprises from different fields are taking advantage of its ability to simulate real-time working conditions and perform intelligent decision-making, where a cost-effective solution can be readily delivered to meet individual stakeholder demands. Most approaches today lack a comprehensive review to examine Digital Twin benefits by considering both engineering product lifecycle management and business innovation as a whole. The systematic review further identifies eight future perspectives for Digital Twin, including modular Digital Twin, modeling consistency and accuracy, incorporation of Big Data analytics in Digital Twin models, Digital Twin simulation improvements, VR integration into Digital Twin, expansion of Digital Twin domains, efficient mapping of cyber-physical data and cloud/edge computing integration [12].

Factory planning is a complex process chain which involve several teams and individuals. The collaboration of various persons with different points of view can lead to conflicts and misunderstandings due to, for instance, differences among domains' 
vocabulary. One solution to solve this problem is to formalize the technical terms of each domain and to create correspondences among concepts defined within each domain. This can be achieved by defining an ontology of the domains. An ontology allows to represent concepts and relationships between these concepts that can be used to both infer implicit knowledge and to check the consistency of the domain definition by resorting to an inference engine [13].

Beside collaboration, another key factor in modern product design is the ability to reuse existing knowledge in design, products or processes. In this scope, one major change during the last years is the democratisation of Knowledge-Based Engineering (KBE). KBE is a large field at the crossroads of Computer-Aided Design (CAD), process planning, factory planning, artificial intelligence and programming [13]. A cross-domain, downstream approach promises most benefits.

Knowledge-based engineering templates are intelligent models or features that are able to store design intent as well as product and process knowledge. This can then restitute the knowledge by adapting them to design contexts, i. e. environments where the template is used, such as a car assembly model or a wing model. The following terms are often used: intelligent template or object-oriented template [13]. Templates can be used both for the automation and synchronization of processes.

A process template represents a structured sequence of tasks related to the CADsystem elements based on the underlying business process which significantly exceeds the border of the design process. A CAD-based process template controls the information flow between the CAD elements and triggers multiple actions based on user interaction or dedicated events, e. g. instantiation of other templates. A process chain template extends this scope to connect different domains (e. g. design to manufacturing) which is important in context of Digital Twin [14]. A template is able to store comprehensive, complete information about the evolution of the formal problem modeling and the corresponding auxilary data.

Templates can be used in the assembly modeling of complex products, where it is not unusual facing an explosive growth of assembly relations, or called combination explosion. Through integrating object-oriented methods with the knowledge template based modeling, it is possible to deal with the complexity of the assemblies of large-scale products. As such, the templates with various assembly relations can be defined for complex products; constitutive parts or subassemblies in a product structure are defined as object classes; their features can be encapsulated, and defined or modified freely via the parameters of object classes to meet the requirements of a specific instance. This method supports reusing knowledge and simplifying assembly modeling of complex products such as aero-engines [15].

The generation and instantitation of intelligent process templates set several strict pre-requisites to process constituents: clear process definition [16], proper data structure [17], high data quality [18], high-level interface [19], deep modularization [20], and a stable and reliable control environment [21]. Furthermore, a template must be maintained over a long period of time and can become subject of intellectual property theft [22]. The main gains of template use are the process acceleration, lower operating costs, improved traceability and error avoidance.

A particular challenge consists of generation of a Digital Twin in a built environment where the necessary data are not available and must be acquired either from physical object or analog documents. With the regard to a previous publication [23], where the approach for automated object recognition is described, we will concentrate here to the 
last step: acquisition of models in the planning step by using a high-level process automation by intelligent workflow.

\section{Generation of a Digital Twin}

To generate a Digital Twin as automatically as possible, the method presented here applies fast scans of the shop floor and subsequent object recognition using artificial intelligence algorithms. Here, the production layout (e. g. size and location of objects) and production logics, such as machine types and transport routes, are captured automatically and mapped to scale in digital simulation models. For efficiently generating a Digital Twin, three different input sources can be distinguished, as shown in Figure 1 [24]:

- Group A parameters is obtained directly from a scan (e. g. machine geometries). Different scanning methods, such as laser scanning or photogrammetry, are utilized here.

- Group B parameters require additional object recognition (e. g. machine types). To record these parameters, a reference database of Computer-Aided Design (CAD) models is necessary in addition to the production scan. A matching of the scanned object with the CAD models of the reference database provides the required input parameters for the Digital Twin.

- Group C parameters, contrary to the others, cannot be captured generically or automatically. They are determined on a company-specific basis (e. g. machine times). Appropriate forms are developed for the acquisition of this parameter group, so that a survey of typical parameters can be carried out and this process can be performed with the least possible effort.

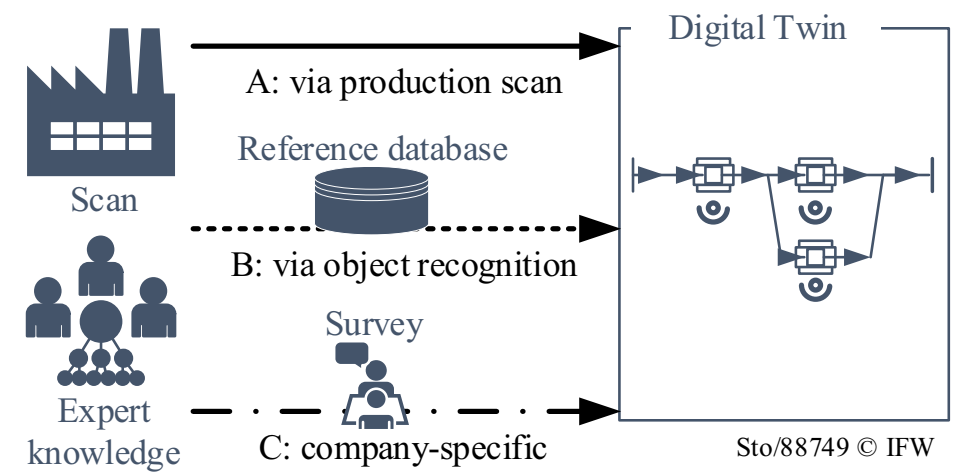

Figure 1: Overview of the automated generation of the Digital Twin [24]

For the defined parameter groups, specific data is required. The parameter definition is derived from factory planning, especially material flow planning, investment planning, and capacity planning, in order to consider their requirements and to narrow down the subject matter. Thus, long-term planning processes and analyses of the existing system are both considered, this wide-raging selection is suitable as a basis to generally determine the required parameters. Objects such as machine tools, workstations, workers, conveyors, and load carriers must be built into the simulation model. In order to 
recognize and create these objects sufficiently for the Digital Twin, the three parameter groups mentioned above are used. For each object, there are different parameters that are relevant for the simulation model. This will be shown in more detail in the following example.

First, the parameters that can be obtained directly by the scanning process are determined. For the object "machine tools", these parameters consist mainly of the spatial information. Since the simulation model is not able to process detailed CAD data, the dimensions of the bounding box are used as an acceptable simplification. Further useful parameters are spatial relations to other objects of the production system.

For the second group of parameters an object detection is connected. By a similarity analysis of the spatial information with an external database, further information can be added to the model. Object recognition makes the stored information available for the Digital Twin. For the example of machine tools, different data can thus be provided. In general, all generic properties of machine tools are conceivable, such as power, machining area or capacity. This procedure can be applied to machine tools, as well as to other objects, such as workstations or transport equipment, to store comprehensive information in the external database and thus provide it to the Digital Twin.

The third parameter group, the company-specific data, cannot be captured with a scan and subsequent object recognition. For the consideration of machine tools, this group includes: The link logic to upstream and downstream processes or machines, processing, setup, distribution and recovery times, setup information, downtimes and their distribution, shift calendars and availability times. Additionally, specifics of the respective simulation software may have to be determined, e. g. classifications of the resource type or reference periods for statistical recording. While the first two parameter groups can be recorded nearly automatically, the third group requires manual effort. By developing forms and interviews, this process can still be made efficient. It is also examined to what extent standardized interfaces to IT systems can be pre-defined in order to access company-specific data.

A total of 321 parameters for the Digital Twin were identified and a parameter group was assigned to each of them. In addition, a description of the parameter, a label for the data transmission, whether it is a mandatory entry, a data type, the requirement for updating or continuous recording and an assignment to the described use cases were compiled for documentation purposes. With this, it is possible to develop a data interface to a simulation software to automatically generate a model, which will be further described in the following.

The approach for an automatic generation of a Digital Twin consists of three fundamental steps:

1. Scanning the production system to obtain a point cloud

2. Modelling with the objective of creating a mock-up

3. Simulation modelling for the generation of a Digital Twin

Figure 2 shows an overview of the approach by integrating the three steps mentioned above into a data model. Here it becomes apparent how the three parameter groups relate to the three process steps. A detailed description can be found in [24]. For step 1 a detailed point cloud can be generated by laser scanning. Here, the further processing through object recognition and the subsequent modelling up to the Digital Twin gains a sufficient basis. Further information on the scanning process was published in [23]. The modelling, step 2, needs to be heavily supported by object recognition to save time and effort. To achieve this, a database is required, in which CAD models of suspected objects 
are stored. Scalability is an important requirement for this approach, because theoretically each of the infinite built objects needs to be recognised. Further object parameters are stored in an external database and provided for the last step. The technical procedure and a description of the algorithms used are presented in [25]. In step 3 this information is used for the simulation modelling of the Digital Twin. An interface is used in order to retain the class structure when transferring information from CAD to the simulation software. The expert knowledge and the company-specific information of the production system need to be acquired by forms or expert interviews and inserted in the simulation modelling process. It is also conceivable that data could be taken directly from IT systems. This third step will be discussed in more detail below.

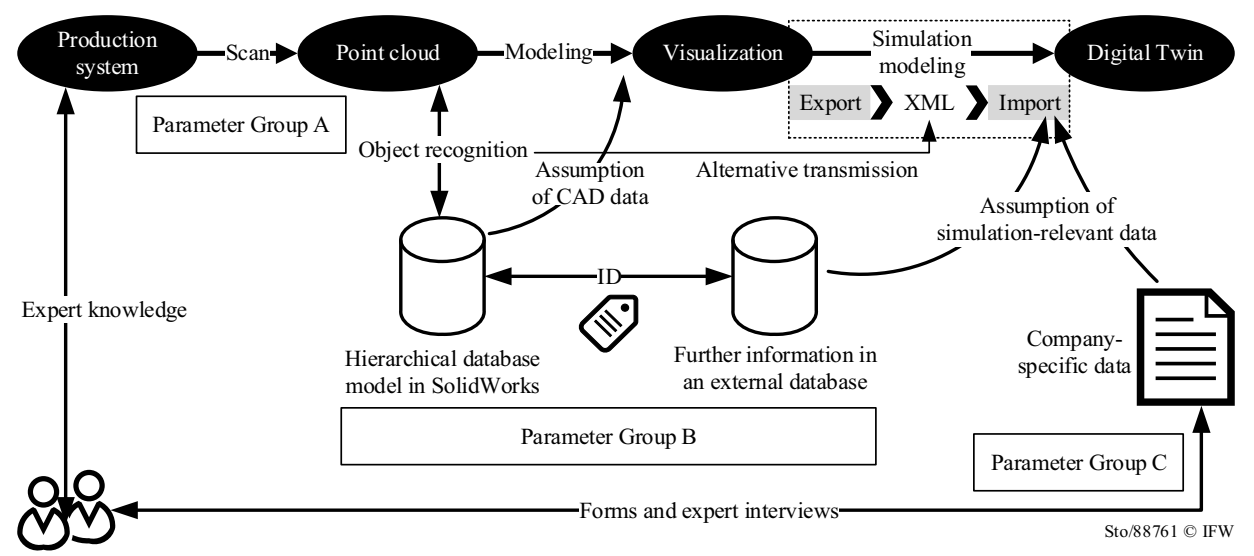

Figure 2: Overview of the overall process

\section{Simulation modelling}

The simulation modelling receives as an input the model data from the object recognition, in particular the spatial information of the object as well as the specific properties of the objects. For this purpose, a format is required that distinguishes between objects, that means that it is object-oriented, platform-independent, and is open-source, so that an adaptation to the concrete requirements can be carried out. This format is provided by the XML schema and is therefore used for the project [26]. Accordingly, the input is transmitted in an XML file.

Two cases can be distinguished here: On the one hand, the XML file can be generated directly from the object recognition process. This approach is applied in the project, because it allows to achieve the highest performance, without additional manual affort, and to avoid transmission errors. On the other hand, the XML file can also be generated by exporting the CAD model. This approach was also developed in the project, but at this point there remain the mentioned performance and transmission problems. However, this second procedure still has its justification, since the simulation modelling can be used with already existing CAD models.

$\mathrm{XML}$ is a format that allows data to be stored in text files in a structured manner [26]. In the present case the XML format was used as CAEX. This format specification provides requirements for the structure of the XML file. For the presented each data item of the XML file represent an object in the model. Here a distinction is made between the 
input parameters already described, such as machines, conveyors or transport equipment. Every item is assigned attributes. These attributes can be different for various objects, depending on the properties of the objects. For example, processing time can be stored for machines, while for a conveyor belt the length is stored in the XML file. However, it is also possible to store the same attributes for both example objects mentioned. For example, the locations in the model are transferred here in $\mathrm{X}$ and $\mathrm{Y}$ coordinates.

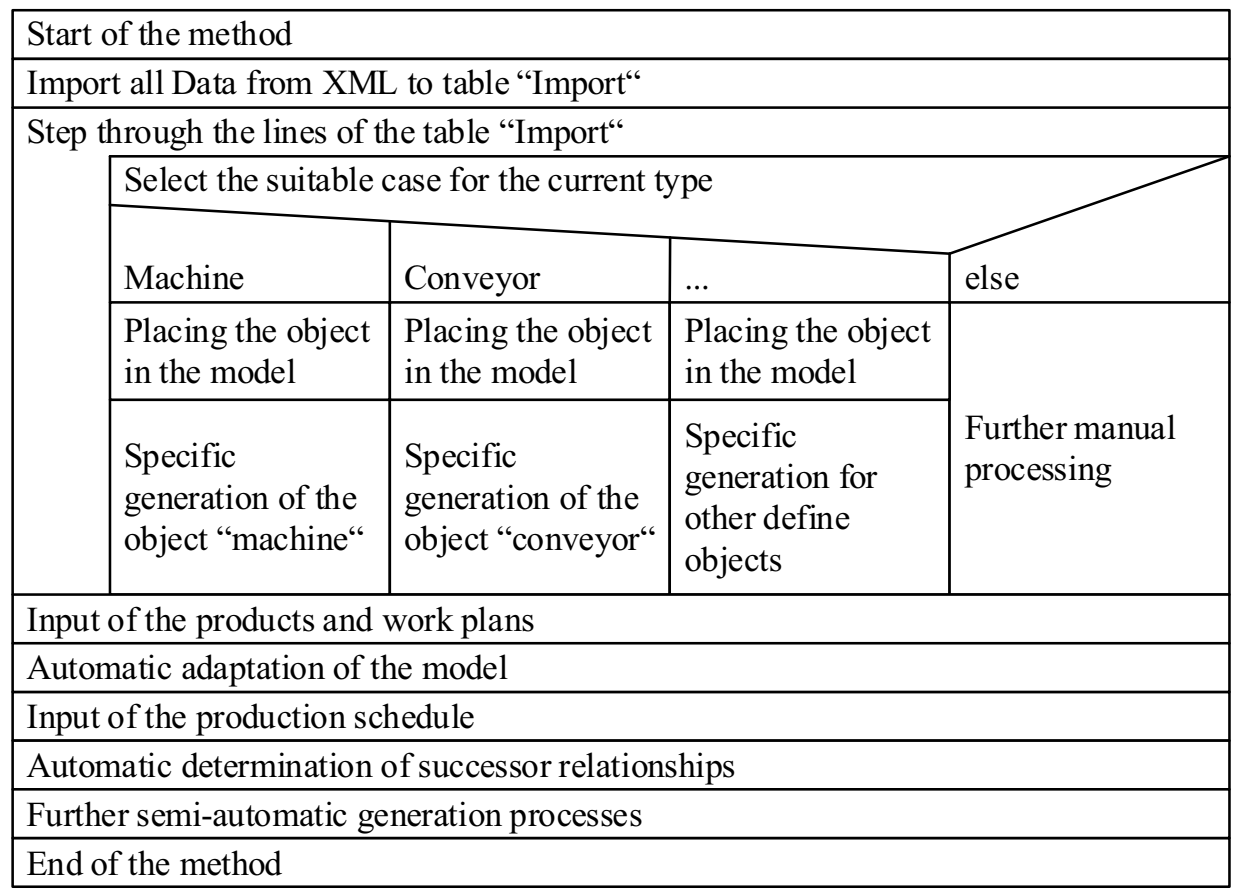

Figure 3: Representation of the simulation modelling process with a Nassi-Shneiderman diagram

The import of the XML file to the simulation software is structured in such a way that all values from the XML file are first written into an internal table of the simulation software. This procedure is useful because it reduces the calculation time. In the program code, this import is autarkic to the model creation. The internal table in the simulation software corresponds to the structure of the XML file, i.e. all attributes correspond to columns and the single objects are listed line by line.

The model construction is carried out by an autarkic method, too. For this, the internal table created by the import is step by step run through. Each line corresponds to an object of the model, so that a simulation module is generated here in each case. In the first step, a case analysis is carried out, which depends on which object type is given. This means that a different generation process is carried out for a machine object type than for a means of conveyor, for example. This corresponds to the differently stored parameters, as mentioned above. In a second step, a part of the model is then generated for each object. For this purpose, the item data is first used to place the object. This data must be scaled to the size of the simulation model surface. Then the specific data is added to the generated module. As already mentioned, these differ depending on the type of object. 
Once the method has been completed, a rudimentary model is obtained. User input or a export from other IT-Systems, for example from a manufacturing execution system (MES), is required for further automated generation steps. For example, an additional method has been developed that implements predecessor and successor relationships in the model based on the production schedule. For this, however, the production schedule is required first, which cannot be determined by the scan and the subsequent object recognition. In order to design this process as efficiently as possible, predefined tables have been stored in the model, which can be filled either with minimum effort by experts of the production system or by a defined export. Figure 3 summarises the process again. By the end of the method or the connected methods after user input, a conditionally operable simulation model is obtained, which can be extended to a Digital Twin.

After the simulation model has been built, factory planning measures can be applied. In the approach presented here, these are also predefined and can be executed with a minimum of manual effort. For example, the value stream of the products can be displayed directly in a Sankey diagram. In this case, only the interpretation by experts is an additional manual effort. Another example is the bottleneck analysis, which can also be performed automatically. Here the bottleneck station is identified by considering the proportion of value added in relation to the waiting proportion. These values are generated by a simulation run. In this context, further examples of measures in factory planning are investment planning, inventory management and layout planning.

\section{Results}

The procedure shown was applied in an example scenario. The following section summarizes the most important findings.

The export method, with which the creation of the XML file can be performed automatically, was programmed in a macro for SolidWorks. It is open source and can be therefore downloaded from [27]. The macro is written with the Visual Basic for Applications (VBA) programming language and must be triggered manually in SolidWorks while a model is open. The XML file is the direct result of the macro. An XML file in CAEX format is available, which contains the objects of the model and their attributes as explained. The import was implemented in the software Plant Simulation. Here an internal program function was used to transfer the internal table with the data from the XML file. Such predefined commands are often found in other simulation software, which again emphasizes the advantage of using XML. The program code for generating the model is also published and can be found in [28].

As only one example scenario has been carried out at present, it is not possible to specify what effort can be saved by the method. However, it can be concluded that the effort for scanning the production hall is additional. This can take one to two working days, depending on the size of the hall. The subsequent object recognition, the generation of the CAD model, the export from the CAD software respectively the import into the simulation software and the first rudimentary model generation can be automated and therefore carried out with a minimum of effort. The subsequent finalization of the simulation model is supported by further automation and predefined templates respectively interfaces. Therefore, considerable cost savings can be expected here, which more than offset the additional effort for scanning. 


\section{Conclusions and Outlook}

The article presented here has shown how the generation of a Digital Twin can be achieved efficiently and with several automated steps. It was explained how required input parameters were derived from the targeted use cases. Afterwards the whole process was explained in detail. This novel process reduces the effort to generate a Digital Twin, so that the utilization can be beneficial for a multitude of companies, in particular SMEs.

Our focus was set on three of the aforementioned eight future perspectives for Digital Twin: modular Digital Twin, modeling consistency and accuracy, and Digital Twin simulation improvements. At first, we have divided the DT into several modules according to the factory structure, thereby achieving greater interchangeability and repeatability.

By generating and using pre-made CAD models of the machines and the devices from a library, the highest requirements for model consistency, quality and accuracy could be met, which are comparable with the remastering of the CAD models. Furthermore, this approach opens the door for a cooperation with the manufacturers of the factory equipment and the vendors of object libraries as well.

The use of intelligent templates on the one hand achieved a high degree of automation and on the other hand created the possibility of expanding the simulation environment (e.g. ergonomic studies).

Finally, a clear process definition combined with a high-level interface facilitates a stable and reliable control environment. Based on an highly accurate object recognition, our approach provides a significant process acceleration, improved traceability and error avoidance at lower operating costs.

In addition to the technical extensions, the procedure must be validated in further applications. Only then can it be finally determined how much effort can be saved by applying the procedure.

\section{Acknowledgement}

The authors gratefully acknowledge, that the presented method is a result of the research project "DigiTwin - Efficient generation of a Digital Twin for manufacturing" which is granted by the Federal Ministry of Education and Research (BMBF) under the funding measure "KMU-innovativ: Produktionsforschung" (funding code 02K18K003).

\section{References}

[1] S. Bierschenk, A. Ritter, T. Kuhlmann, Stand der Digitalen Fabrik bei kleinen und mittelständischen Unternehmen. Studie, Fraunhofer, Stuttgart, 2005.

[2] S. Wenzel and T. Peter, Prozess-Simulation in kleinen und mittleren Unternehmen mittels des Bausteinkastensystems KMUSimMetall. In: S. Wenzel, T. Peter, (Eds.): Simulation in Produktion und Logistik, University Press, Kassel, 2017, pp. 433-442.

[3] S. Spieckermann, Diskrete, ereignisorientierte Simulation in Produktion und Logistik Herausforderungen und Trends, Simulation und Visualisierung 2005 - SimVis. SCS Publishing House, Erlangen, 2005, pp. 3-14.

[4] M. Rabe, S. Spieckermann, S. Wenzel, Verifikation und Validierung für die Simulation in Produktion und Logistik: Vorgehensmodelle und Techniken, Springer-Verlag, Berlin, 2008.

[5] S. Wenzel, M. Weiß, S. Collisi-Böhmer, H. Pitsch, O. Rose, Qualitätskriterien für die Simulation in Produktion und Logistik. Springer, Berlin, 2008. 
[6] P. Nyhuis, H.-P. Wiendahl, Logistische Kennlinien: Grundlagen, Werkzeuge und Anwendungen, Springer-Verlag, Berlin, 2012.

[7] R.Y. Zhong and W. Ge, Internet of things enabled manufacturing: a review, International Journal of Agile Systems and Management, Vol. 11, 2018, No. 2, pp. 126-154.

[8] J. Bischoff, C. Taphorn, D. Wolter, N. Braun, M. Fellbaum, A.Goloverov, S. Ludwig, T. Hegmanns, C. Prasse, M. Henke, M. ten Hompel, F. Döbbeler, E. Fuss, C. Kirsch, B. Mättig, S. Braun, M. Guth, M. Kaspers and D. Scheffler, Erschließung der Potenziale der Anwendungen von „Industrie 4.0 “ im Mittelstand. Fraunhofer, Dortmund, 2015, http://publica.fraunhofer.de/documents/N-356542.html, accessed Jan 202019.

[9] B. Denkena, F. Winter, S. Hauck, Anlernen von ablauforientierten Simulationsmodellen. ProductivITy, Vol. 21(3), 2016, pp. 52-54.

[10] Weissman, A.; Wegerer, S.: Unternehmen 4.0: Wie Digitalisierung Unternehmen \& Management verändert. In: Erner, M. (Ed.): Management 4.0. Berlin: Springer 2019, pp. 43-78.

[11] A. J. H. Redelinghuys, A. H. Basson, K. Kruger, A six-layer architecture for the digital twin: a manufacturing case study implementation, Journal of Intelligent Manufacturing, 2019, https://doi.org/10.1007/s10845-019-01516-6.

[12] K.Y. Hong Lim, P. Zheng and C.-H. Chen, A state-of-the-art survey of Digital Twin: techniques, engineering product lifecycle management and business innovation perspectives, Journal of Intelligent Manufacturing, 2019, https://doi.org/10.1007/s10845-019-01512-w.

[13] O. Kuhn, H. Liese and J. Stjepandic, Methodology for knowledge-based engineering template update, IFIP Advances in Information and Communication Technology, Vol. 355, Springer, Berlin Heidelberg, 2011, pp. 178-191.

[14] J. Stjepandić, W.J.C. Verhagen, H. Liese and P. Bermell-Garcia, Knowledge-based Engineering, in: J. Stjepandić et al. (eds.) Concurrent Engineering in the 21st Century: Foundations, Developments and Challenges, Springer International Publishing Switzerland, 2015, pp. 255-286.

[15] L.D. Xu, C. Wang, Z. Bi and J. Yu, Object-Oriented Templates for Automated Assembly Planning of Complex Products, IEEE Transactions on Automation Science and Engineering, Vol. 11, Issue 2, April 2014, pp. $492-503$.

[16] R.C. Beckett, Functional system maps as boundary objects in complex system development, International Journal of Agile Systems and Management, Vol. 8, 2015, No. 1, pp. 53-69.

[17] A. Biahmou, C. Emmer, A. Pfouga and J. Stjepandić, Digital master as an enabler for industry 4.0, Advances in Transdisciplinary Engineering, Vol. 4, 2016, pp. 672-681.

[18] S. Bondar, C. Ruppert and J. Stjepandić, Ensuring data quality beyond change management in virtual enterprise, International Journal of Agile Systems and Management, Vol. 7, 2014, Nos. 3/4, pp. 304323.

[19] C. Emmer, A. Fröhlich and J. Stjepandić, Advanced engineering visualization with standardized 3D formats, IFIP Advances in Information and Communication Technology, Vol. 409, Springer, Berlin Heidelberg, 2013, pp. 584-595.

[20] E. Ostrosi, J. Stjepandić, S. Fukuda and M. Kurth, Modularity: New trends for product platform strategy support in concurrent engineering, Advances in Transdisciplinary Engineering, Vol. 1, 2014, pp. 414-423, DOI: 10.3233/978-1-61499-440-4-414.

[21] J. Ríos, F.Mas Morate, M. Oliva and J.C. Hernández, Framework to support the aircraft digital counterpart concept with an industrial design view, International Journal of Agile Systems and Management, Vol. 9, 2016, No. 3, pp. 212-231.

[22] H. Liese, J. Stjepandić and S. Rulhoff, Securing product know-how by embedding IP-protection into the organisation, 2010 IEEE International Technology Management Conference, ICE 2010, 7477025.

[23] M. Sommer, J. Stjepandić, S. Stobrawa and M. von Soden, Automatic Generation of Digital Twin Based on Scanning and Object Recognition, Advances in Transdisciplinary Engineering, Vol. 10, 2019 , pp. 645-654.

[24] B. Denkena, M.A. Dittrich, S. Stobrawa and J. Stjepandic, Automated Generation of a Digital Twin Using Scan and Object Detection for Data Acquisition, Simulation in Produktion und Logistik 2019, Wissenschaftliche Scripten. Auerbach, 2019, pp. 49-58.

[25] Salem B, Stjepandic J, Stobrawa S. Assessment of Methods for Industrial Indoor Object Recognition. Transdisciplinary Engineering for Complex Socio-technical Systems, Proceedings of the 26th ISPE Inc. International Conference on Transdisciplinary Engineering, IOS Press, Tokyo: 2019, p. 390-399.

[26] C. M. Chituc, XML interoperability standards for seamless communication: An analysis of industryneutral and domain-specific initiatives, Computers in Industry, Vol. 92-93, 2017, pp. 118-136.

[27] Stobrawa S. SolidWorks Export-XML IFW DigiTwin. DOI: 10.5281/zenodo.3629369 downloadable under https://zenodo.org/badge/latestdoi/236736366

[28] Stobrawa S. PlantSimulation Import \& ModellGeneration IFW DigiTwin. DOI: 10.5281/zenodo.3618211 downloadable under https://zenodo.org/badge/latestdoi/235356220 